03

\title{
Изменение потенциала пространства в плазме тлеющего разряда при движении ударной волны
}

\author{
(C) А.С. Барышников, И.В. Басаргин, Н.О. Безверхний, С.В. Бобашев, Н.А. Монахов, П.А. Попов, \\ В.А. Сахаров, М.В. Чистякова
}

Физико-технический институт им. А.Ф. Иофрфе РАН, 194021 Санкт-Петербург, Россия

e-mail: nm1988@mail.ru

Поступило в Редакцию 4 марта 2019 г.

В окончательной редакции 4 марта 2019 г.

Принято к публикации 11 марта 2019 г.

\begin{abstract}
В экспериментах по взаимодействию ударной волны с тлеющим разрядом установлено, что движение ударной волны сопровождается увеличением потенциала плазмы разряда. Перед фронтом ударной волны наблюдается сравнительно малое изменение потенциала, а за фронтом - существенное. Меняющийся потенциал в плазме тлеющего разряда вызывает искажение тока двойного зонда. Предложен метод его коррекции, заключающийся в совместной обработке двух сигналов, полученных в отдельных опытах с различной площадью поверхности отрицательного электрода зонда. Метод позволяет определить ионный ток и изменение потенциала пространства. Ионный ток, определяемый концентрацией заряженных частиц, до прихода ударной волны в точку измерения остается неизменным. За фронтом волны наблюдается плавное увеличение ионного тока в отличие от скачкообразного изменения газодинамических параметров на фронте волны.
\end{abstract}

Ключевые слова: тлеющий разряд, ударная волна, низкотемпературная плазма, электрический зонд.

DOI: 10.21883/JTF.2019.10.48167.81-19

\section{Введение}

Зондовый метод является одним из основных методов диагностики низкотемпературной плазмы [1-7]. В частности, широко используется двойной электрический зонд, представляющий собой два электрода, между которыми приложено постоянное напряжение. При достаточной величине этого напряжения двойной зонд находится в режиме насыщения ионного тока, при котором протекающий ток определяется только концентрацией ионов в окрестности отрицательного электрода. Этот инструмент является простым и удобным в исследованиях плазмы неограниченного объема, например, ионосферы [2], или при изучении безэлектродных высокочастотных разрядов в камерах с изолирующими стенками [8-10]. Одним из преимуществ таких измерений является то, что измерительная система находится под плавающим потенциалом относительно плазмы, и это позволяет использовать двойной зонд для измерений в нестационарных процессах. Еще одно важное преимущество заключается в малости возмущений, вносимых двойным зондом в плазму, поскольку ток в его цепи всегда ограничен по величине ионным током насыщения.

Несмотря на перечисленные достоинства двойного зонда, измерения в плазме электродного разряда осложняются наличием гальванической связи между зондом и электродами разрядного промежутка. Так, при изменении потенциала плазмы результаты измерений могут быть искажены переходным процессом в измерительной цепи. С данной проблемой авторы столкнулись при изучении взаимодействия поперечного тлеющего разряда с ударной волной [11-15]. Авторами этих работ обнаружено, в частности, что движение ударной волны в плазме разряда сопровождается изменением тока двойного зонда до прихода ударной волны в точку измерения. Решению этой задачи посвящена настоящая работа.

\section{Экспериментальная установка и техника измерений}

Эксперименты проводились на плазмогазодинамической установке в воздушной среде при давлении $4 \mathrm{kPa}$. Схема установки показана на рис. 1. В рабочей каме-

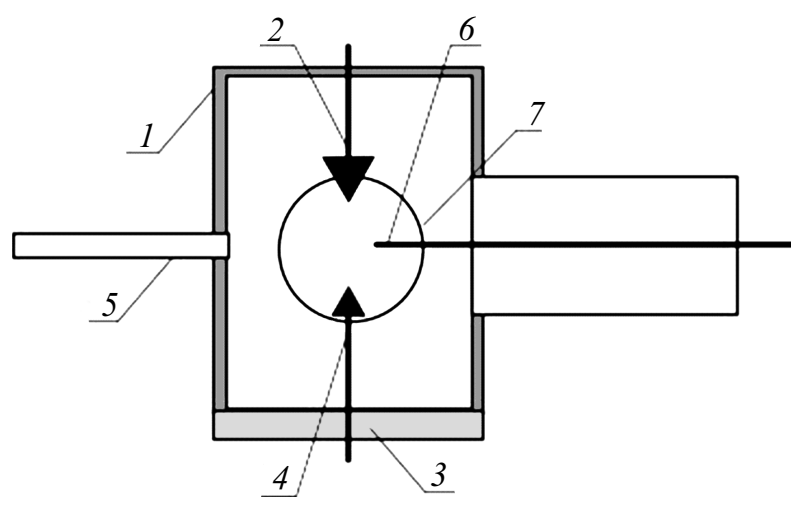

Рис. 1. Схема установки: $1-$ рабочая камера, $2-$ катод, 3 - изолирующая пластина, 4 - анод, 5 - электроразрядная ударная труба, 6 - двойной зонд, 7 - наблюдательное окно. 
pe 1 внутренним диаметром $300 \mathrm{~mm}$ и высотой $400 \mathrm{~mm}$ создавался стационарный тлеющий разряд между двумя 60-градусными коническими медными электродами, расположенными на расстояние $100 \mathrm{~mm}$. Постоянное горение разряда поддерживается источником напряжения, обеспечивающим протекание тока силой $1 \mathrm{~A}$ при напряжении на разрядном промежутке $680 \mathrm{~V}$. Рабочая камера 1 и катод 2 имеют нулевой потенциал. Анод 4 расположен на непроводящей пластине 3 , закрывающей нижний торец рабочей камеры. Взаимное расположение электродов (анод внизу, катод сверху) и их форма продиктованы необходимостью пространственной стабилизации разряда.

Ударная волна формируется электроразрядной ударной трубой 5 с внутренним диаметром $30 \mathrm{~mm}$ и длиной $700 \mathrm{~mm}$. Выходной торец ударной трубы расположен заподлицо с боковой стенкой рабочей камеры. Скорость ударной волны на выходе ударной трубы составляет $1.3 \mathrm{~km} / \mathrm{s}$. Ось ударной трубы проходит посередине разрядного промежутка. Начальное давление в канале ударной трубы равно давлению в рабочей камере.

Двойной электрический зонд 6 перемещается в горизонтальнаой плоскости соосно с ударной трубой. Зонд представляет собой два платиновых электрода диаметром $0.5 \mathrm{~mm}$ и длиной $10 \mathrm{~mm}$, расположенных параллельно друг другу на расстоянии $8 \mathrm{~mm}$. Между электродами двойного зонда приложено постоянное напряжение, обеспечивающее работу зонда в режиме насыщения ионного тока.

Регистрация пространственного положения ударной волны и запуск системы регистрации осуществляются с помощью двух оптических шлирен-систем через два окна 7 на боковой поверхности рабочей камеры. Луч одной оптической системы неподвижен и расположен у левого края наблюдательного окна. С ее помощью осуществляется запуск системы регистрации. Луч второй системы перемещается вместе с зондом, с помощью которой регистрируется момент прихода ударной волны в точку измерения. Погрешность измерения положения ударной волны составляет не более $3 \mathrm{~mm}$.

\section{Результаты измерений и их обсуждение}

На рис. 2 показано изображение тлеющего разряда, который имеет форму тела вращения. Интенсивность свечения разряда максимальна в центре и плавно уменьшается к периферии. На вершине нижнего конуса (анода) наблюдается яркое пятно, где происходит контракция разрядного тока. В непосредственной близости от поверхности катода (верхний конический электрод) видна яркая область, занимающая сравнительно небольшую часть поверхности катода. Это катодное свечение характерная особенность тлеющего разряда.

Направление, вдоль которого перемещается двойной зонд, показано на рис. 2 штриховой линией. Ударная волна движется слева направо по направлению оси $X$, а зонд

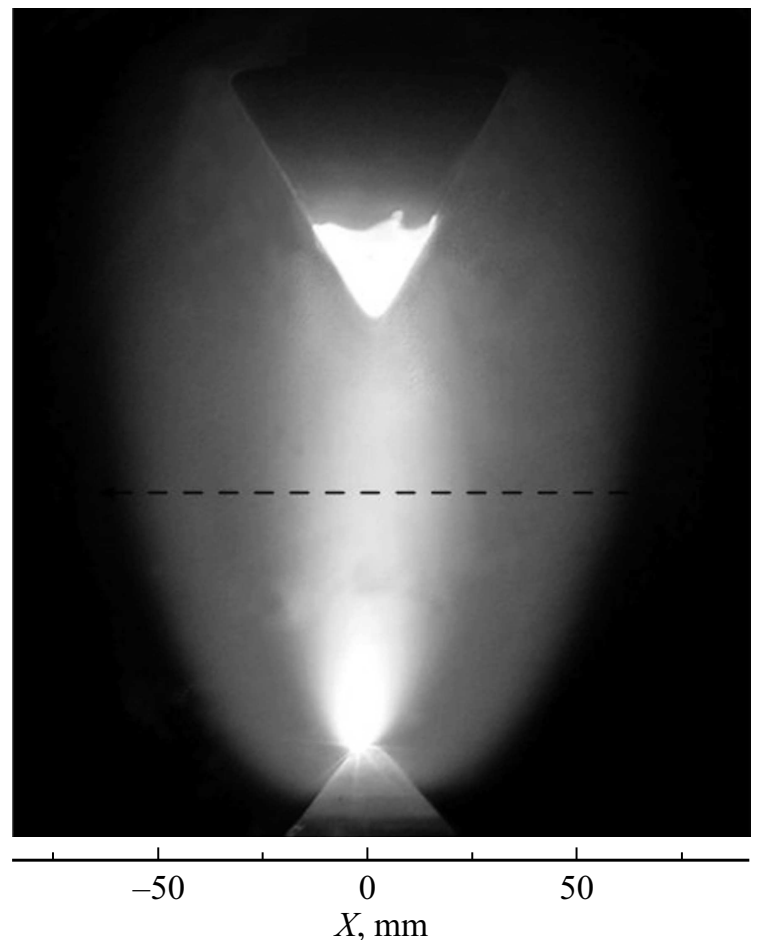

Рис. 2. Внешний вид тлеющего разряда.

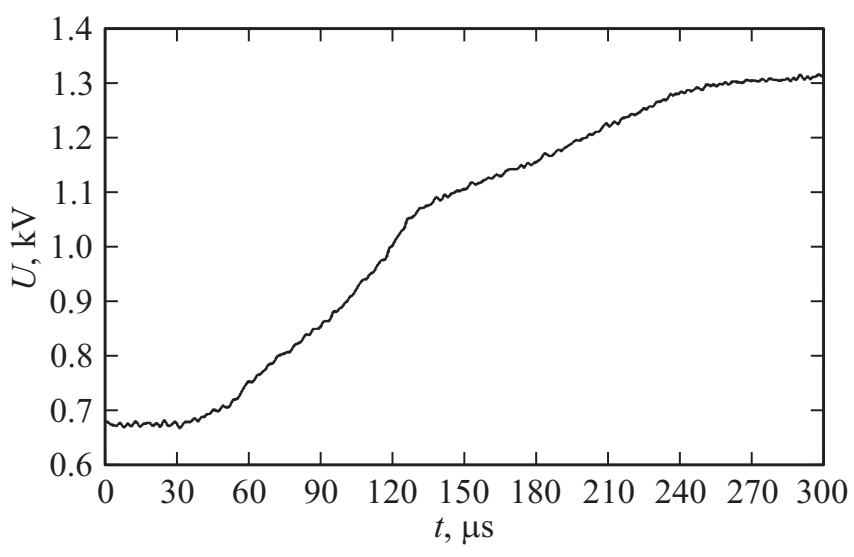

Рис. 3. Изменение напряжения на аноде разрядного промежутка при движении ударной волны в плазме тлеющего разряда.

располагается справа от оси разряда. Положение зонда справа от оси позволяет изучать плазму в пространстве перед фронтом ударной волны.

Движение ударной волны сопровождается изменением электрических параметров тлеющего разряда, в частности, напряжения на аноде разрядного промежутка. На рис. 3 показано изменение анодного напряжения при движении ударной волны. Здесь и далее отсчет времени ведется от момента появления ударной волны в области наблюдения. Видно, что после $30 \mu \mathrm{s}$ напряжение монотонно возрастает. В этот момент времени ударная волна находится вблизи левой границы светящейся области 
разряда. В течение времени движения ударной волны по этой области разряда (интервал 50-120 $\mathrm{s}$ на рис. 3) напряжение на разрядном промежутке увеличивается практически линейно.

Увеличение напряжения на разрядном промежутке при движении ударной волной является следствием изменений параметров самой плазмы и, в частности, ее потенциала.

\section{Метод коррекции зондовых измерений}

Электрический зонд, как всякий проводящий элемент, является емкостью относительно заземленного электрода. В плазме разряда емкость зонда заряжена до некоторого напряжения. Из-за наличия гальванической связи между зондом и общим электродом разрядного промежутка при изменении потенциала плазмы возникает ток перезаряда этой емкости.

На рис. 4 показана схема измерений ионного тока двойным зондом и часть вольт-амперной характеристики (BAX) электрического зонда, соответствующая ионной ветви насыщения. Схема измерения состоит из источника постоянного напряжения $E$ и сопротивления нагрузки $R$, которые включены между электродами 1 и 2 двойного зонда. Потенциалы электродов относительно плазмы обозначены $U_{1}$ и $U_{2}$ соответственно. Величина ионного тока $I_{1}$ вычисляется по измеряемому на сопротивлении $R$ напряжению. На схеме символом $C$ обозначена емкость отрицательного электрода 1 двойного зонда.
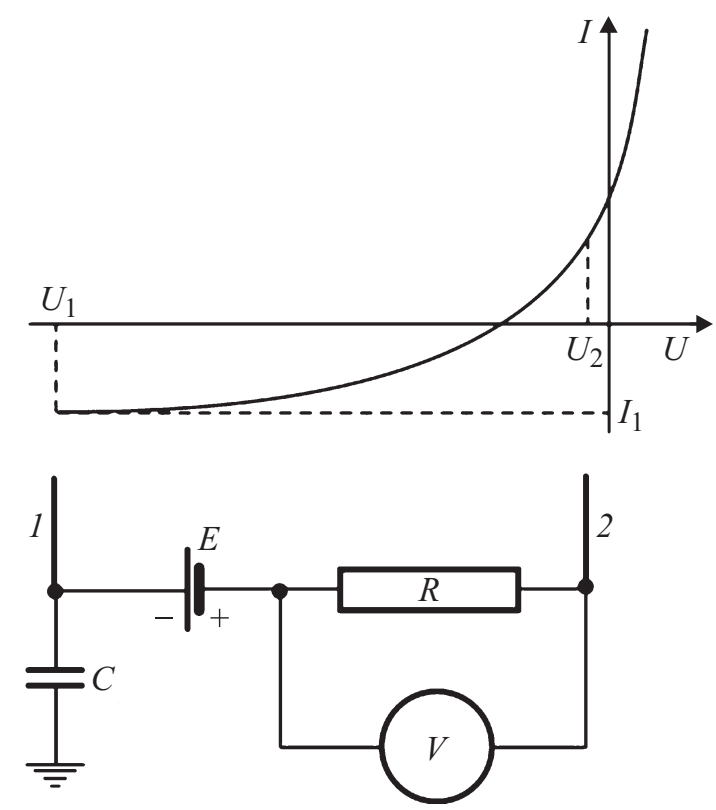

Рис. 4. Схема измерения и ионная ветвь ВАХ электрического зонда: 1,2 - отрицательный и положительный электроды двойного зонда, $E$ - источник напряжения, $R-$ сопротивление нагрузки, $C$ - емкость электрода зонда. $U_{1}$ и $U_{2}-$ потенциалы отрицательного и положительного электродов зонда относительно плазмы, $I_{1}$ - ионный ток двойного зонда.
На ВАХ видно, что потенциалы электродов зонда относительно потенциала пространства разнятся. Так, потенциал отрицательного электрода 1 меньше потенциала пространства на величину, практически равную напряжению между электродами $E$, в то время как потенциал положительного электрода 2 близок к потенциалу плазмы. Именно по этой причине при изменении потенциала плазмы перезаряд емкостей электродов зонда будет происходить через положительный электрод 2. Однако влиять на показание прибора будет только ток перезаряда емкости $C$ отрицательного электрода 2 (она показана на рисунке), поскольку в этом случае ток перезаряда проходит через сопротивление $R$. Таким образом, измеряемый ток зонда определяется суммой тока перезаряда емкости $C$ и ионного тока.

Итак, ток перезаряда емкости зонда проходит через его положительный электрод, а ионный ток определяется параметрами плазмы в окрестности отрицательного электрода зонда. Если при прочих равных условиях изменить площадь поверхности отрицательного электрода зонда, то величина ионного тока измениться пропорционально изменению его площади. При этом ток перезаряда останется неизменным. Имея два измерения, выполненные при разной площади поверхности отрицательного электрода, получим систему двух линейных уравнений

$$
\left\{\begin{array}{l}
I_{i}+I_{C}=I_{1} \\
\alpha I_{i}+I_{C}=I_{2} .
\end{array}\right.
$$

Здесь $I_{i}, I_{C}$ - ионный ток и ток перезаряда емкости, $I_{1}, I_{2}$ - ток зонда, измеренный при разной площади поверхности отрицательного электрода, $\alpha$ - относительное изменение площади поверхности отрицательного электрода. Решение системы (1) имеет вид

$$
I_{i}=\frac{I_{1}-I_{2}}{1-\alpha}, \quad I_{C}=\frac{I_{2}-\alpha I_{1}}{1-\alpha} .
$$

Входящие в (2) величины являются функциями времени за исключением постоянной $\alpha$. Ее значение можно вычислить, взяв во внимание, что в стационарном режиме ток перезаряда емкости отсутствует $I_{C}=0$. В этом случае решение (1) дает значение $\alpha=I_{02} / I_{01}$, равное отношению токов с разной площадью поверхности отрицательного электрода в режиме постоянного горения разряда.

Для определенности примем $\alpha<1$, тогда $I_{1}>I_{2}$. Направление ионного тока не может изменить знак, поскольку оно задано напряжением источника в измерительной цепи. Поэтому решение (2) имеет смысл при выполнении условия $I_{i} \geq 0$. Если это условие нарушено, решение имеет вид $I_{i}=0, I_{C}=I_{1}=I_{2}$.

\section{Результаты обработки данных измерений}

Продемонстрируем возможности предложенного подхода на примере обработки сигналов зонда, получен- 


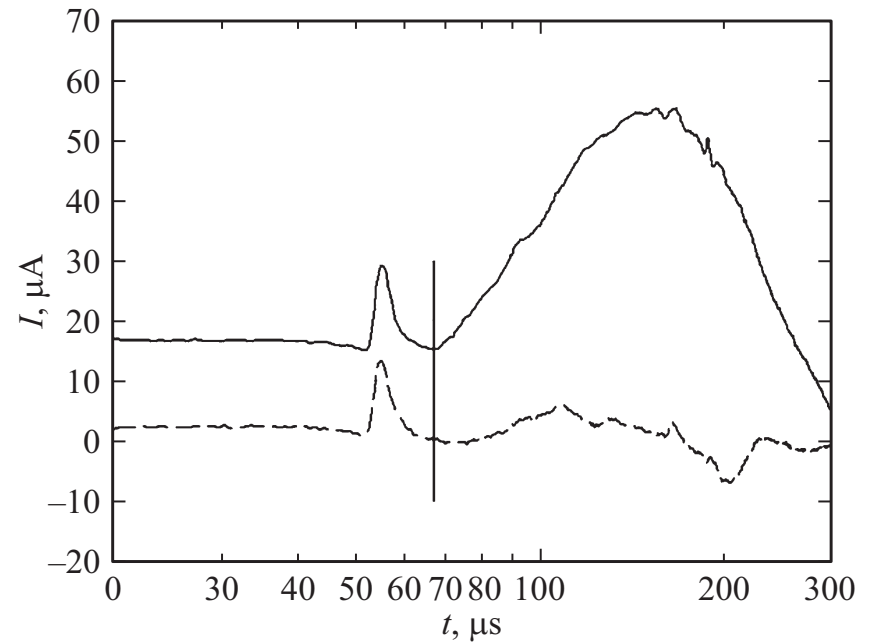

Рис. 5. Измеренные значения тока зонда с открытым (сплошная линия) и частично закрытым (штриховая линия) отрицательным электродом при взаимодействии ударной волны с разрядом. Вертикальная линия - момент прихода ударной волны в точку измерения.

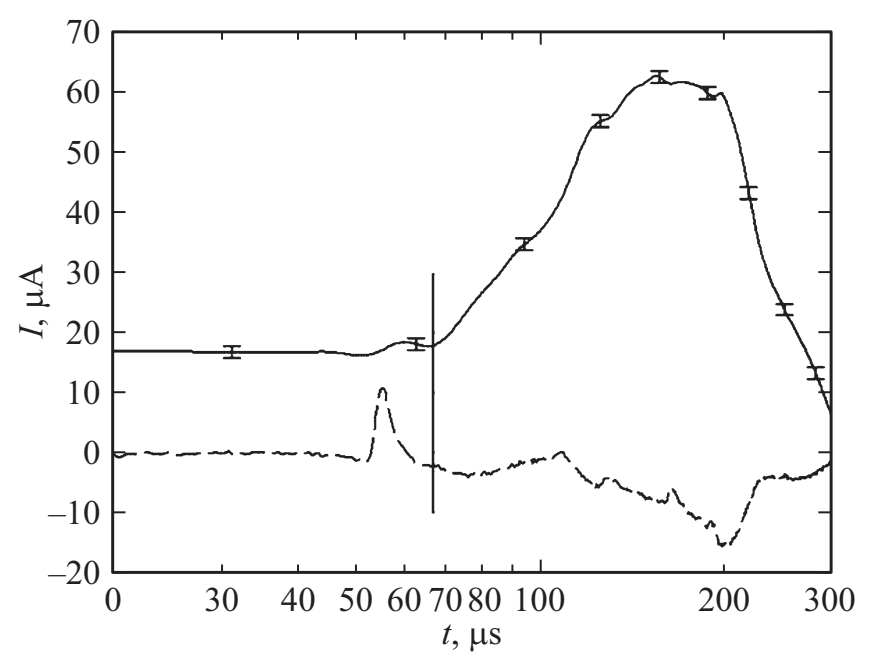

Рис. 6. Рассчитанные значения ионного тока (сплошная линия) и тока перезаряда емкости (штриховая линия) при взаимодействии ударной волны с разрядом. Вертикальная линия момент прихода ударной волны в точку измерения.

ных в упомянутых ранее работах [13-15]. Типичный вид тока зонда показан на рис. 5. Сплошной линией показано изменение тока зонда с открытым электродом, штриховой - изменение тока с уменьшенной площадью поверхности отрицательного электрода зонда. Изменение площади поверхности отрицательного электрода зонда осуществлялось надеванием на него короткого кварцевого капилляра. Момент прихода ударной волны в точку измерения показан на рис. 5 вертикальной линией.

Как уже отмечалось выше, особенностью сигнала зонда является уменьшение тока до прихода ударной волны. На рисунке это наблюдается после $35 \mu \mathrm{s}$ от начала регистрации. В некоторых случаях уменьшение тока составляет более половины начального уровня. Другая особенность сигнала зонда заключается в появлении кратковременного увеличении тока в окрестности момента времени $55 \mu \mathrm{s}$. Исследования показали, что это увеличение тока соответствует прохождению ударной волны центральной части разряда и, по мнению авторов, вызвано взаимодействием ударной волны с областью катодного свечения тлеющего разряда [15].

На рис. 5 видно, что в начале регистрации значения тока не изменяются. Их можно считать равными значениям тока в режиме постоянного горения разряда и использовать для определения величины $\alpha$. Оценки показали, что определенное таким образом значение $\alpha$ соответствует величине относительного изменения площади поверхности отрицательного электрода.

Результаты расчета ионного тока и тока перезаряда емкости зонда, полученные с помощью соотношений (2), показаны на рис. 6. Сплошной линией обозначено изменение ионного тока, штриховой - тока перезаряда емкости зонда. На рис. 6 видно, что до прихода ударной волны в точку измерения ионный ток (сплошная линия) остается неизменным. Наблюдаемые в окрестности момента $55 \mu$ s изменения тока следует считать погрешностью расчета, вызванной недостаточно точным воспроизводством условий эксперимента в разных опытах. За ударной волной наблюдается практически линейное увеличение плотности заряженных частиц в течение примерно $70 \mu \mathrm{s}$. Такое изменение заряженной компоненты отличается от скачкообразного изменения газодинамических параметров на фронте ударной волны [14].

Ток перезаряда емкости зонда (штриховая линия на рис. 6) заметно отличается от нуля примерно после $35 \mu \mathrm{s}$ от начала регистрации. Примерно в этот же момент времени начинается изменение напряжения на аноде разрядного промежутка (рис. 3). На это совпадение следует обратить внимание, поскольку временная корреляция двух параметров, полученных независимыми способами,

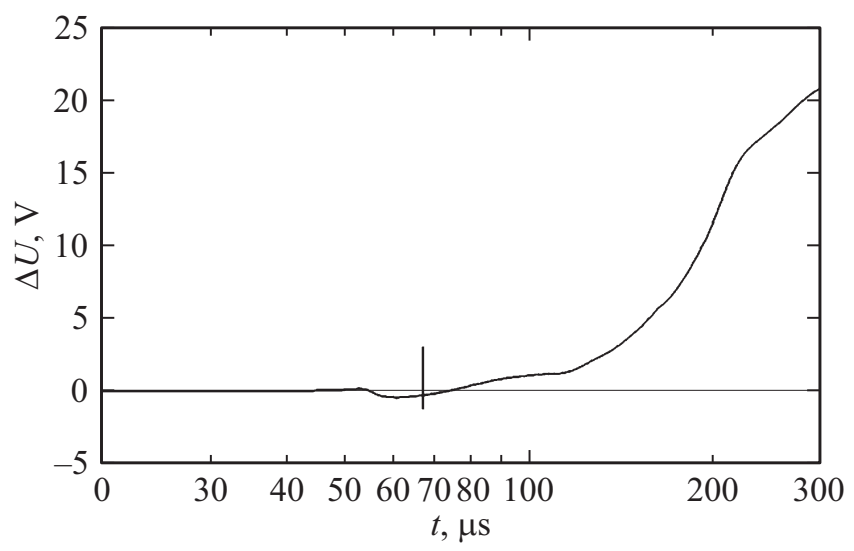

Рис. 7. Изменение потенциала зонда при движении ударной волны в разряде. Вертикальная линия - момент прихода ударной волны в точку измерения. 
говорит об обоснованности предположений, заложенных в методику обработки результатов измерений.

Зная величину емкости зонда $C$, легко рассчитать изменение напряжения на зонде

$$
\Delta U(t)=\frac{1}{C} \int_{0}^{t} I_{C}(\tau) d \tau .
$$

Поскольку причиной возникновения тока перезаряда емкости является изменение потенциала пространства, то естественно связать его с зависимостью (3). Результат интегрирования показан на рис. 7. В расчете использовалось измеренное значение емкости зонда $40 \mathrm{pF}$. На рис. 7 видно, что к моменту прихода ударной волны (вертикальная линия) изменение потенциала составляет доли вольта. Значительное изменение потенциала пространства происходит за фронтом ударной волны примерно после $120 \mu$ s.

\section{Выводы}

Особенность зондовых измерений в плазме электродного разряда связана с наличием гальванической связи между зондом и электродами разрядного промежутка. При изменении потенциала пространства результат измерений искажается током перезаряда емкости зонда по цепи, включающей элементы регистрации ионного тока.

Предложенный метод коррекции результатов измерений заключается в совместной обработке двух сигналов, полученных в отдельных опытах с различной площадью поверхности отрицательного электрода зонда. Метод позволяет определить ионный ток и ток перезаряда емкости зонда. Погрешность метода в основном определяется постоянством воспроизводства начальных условий эксперимента - параметров разряда и интенсивности ударной волны.

В настоящем исследовании установлено, что движение ударной волны в плазме тлеющего разряда сопровождается увеличением потенциала пространства. Начало этого процесса соответствует положению ударной волны вблизи границы светящейся области тлеющего разряда. До прихода ударной волны в точку измерения изменение потенциала сравнительно невелико. Значительное увеличение потенциала пространства происходит за фронтом ударной волны.

Движение волны по центральной части разряда вызывает обратное изменение потенциала пространства, а именно кратковременное его уменьшение, связанное, по-видимому, с воздействием ударной волны на область катодного свечения тлеющего разряда.

Установлено, что отмеченное в ранее опубликованных работах [11-15] изменение тока зонда перед фронтом ударной волны вызвано искажением тока зонда вследствие изменения потенциала пространства. Ионный ток, определяемый концентрацией заряженных частиц, до прихода ударной волны в точку измерения остается неизменным. За фронтом волны наблюдается плавное увеличение ионного тока в отличие от скачкообразного изменения газодинамических параметров на фронте волны.

\section{Конфликт интересов}

Авторы заявляют, что у них нет конфликта интересов.

\section{Список литературы}

[1] Каган В.М., Перель В.И. // УФН. 1963. Т. 81. № 3. C. $409-452$.

[2] Альперт Я.Л., Гуревич А.В., Питаевский Л.П. Искусственные спутники в разреженной плазме. М.: Наука, 1964. $382 \mathrm{c}$.

[3] Swift J.D., Schwar M.J.R. Electrical probes for plasma diagnostics. London: Iliffe books, 1970. 304 p.

[4] Чан П., Талбот Л., Турян К. Электрические зонды в неподвижной и движущейся плазме. М.: Мир, 1978. 204 с.

[5] Демидов В.И., Колоколов Н.Б., Кудрявцев А.А. Зондовые методы исследования низкотемпературной плазмы. М.: Энергоатомиздат, 1996. 240 с.

[6] Chen F.F. // Phys. Plasm. 2001. Vol. 8. N 6. P. 3029-3041.

[7] Райзер Ю.П. Физика газового разряда. Долгопрудный: Интеллект, 2009. 736 с.

[8] Brockhaus A., Korzec D., Werner F. et al. // Surf. Coat. Techl. 1995. Vol. 74. N 1. P. 431-442.

[9] Mildner M., Korzec D., Engemann J. // Surf. Coat. Tech. 1999. Vol. 112. N 1. P. 366-372.

[10] Naz M.Y., Ghaffar A., Rehman N.U. et al. // Prog. Electromagn Res. 2011. Vol. 114. P. 113-128.

[11] Барышников А.С., Басаргин И.В., Бобашев С.В., Монахов Н.А., Попов П.А., Сахаров В.А., Чистякова М.В. // Письма в ЖТФ. 2015. Т. 41. Вып. 15. С. 83-89.

[12] Барышников А.С., Басаргин И.В., Бобамев С.В. и др. // ИФЖ. 2016. Т. 89. № 3. С. 555-558.

[13] Bobashev S.V., Basargin I.V., Baryshnikov A.S. et al. // Proc. of $15^{\text {th }}$ Int. Workshop on Magneto-Plasma Aerodynamics. Moscow, 2016. P. 112-113.

[14] Барышников А.С., Басаргин И.В., Бобашев С.В., Монахов Н.А., Попов П.А., Сахаров В.А., Чистякова М.В. // Письма в ЖТФ. 2017. Т. 43. Вып. 11. С. 20-25.

[15] Baryshnikov A.S., Basargin I.V., Bezverkhnii N.O. et al. // AIAA J. 2018. Vol. 56. N 9. P. 3782-3784. 